\title{
THE RELATIVISTIC ELECTROMAGNETIC STRUCTURE OF THE THREE-BODY SYSTEMS
}

\author{
A.F. Krutov \\ Samara State University, Samara, Russia \\ E-mail: krutovessu.samara.ru
}

M.Yu. Kudinov*

Samara State University, Samara, Russia

E-mail: kudinovessu.samara.ru

\section{V.E. Troitsky}

D.V. Skobeltsyn Institute of Nuclear Physics, Moscow State University, Moscow, Russia

E-mail: troitskyatheory.sinp.msu.ru

In the paper the construction of the electromagnetic current operator for three-body composite system is discussed in the framework of instant form of relativistic quantum mechanics. The construction has performed at the conditions of the current conservation and Lorentz-covariance. The electromagnetic form factor for free system of three fermions with quantum numbers of nucleon is calculated.

The XIXth International Workshop on High Energy Physics and Quantum Field Theory, QFTHEP2010 September 08-15, 2010

Golitsyno, Moscow, Russia

\footnotetext{
* Speaker.
} 


\section{Introduction}

The relativistic description of electromagnetic structure of composite systems is important problem of the nuclear and particle physics over many years. The interest to this problem has increased in connection with recent measurements of electromagnetic and transition form factors of pion and proton $[1,2]$. The relativistic composite models play essential role in the solution of this problem.

One of the main difficulties of the composite model is the construction of conserved and Lorentz-covariant electromagnetic current operator.

In present paper the problem of the construction of the current operator will be considered in the framework of the instant form of the relativistic quantum mechanics (RQM) (see, e.g. [3]). Let us discuss briefly the basic features of this approach.

Wave function in RQM is the solution of eigenvalue problem for complete set of commuting operators. This complete set in the instant form of the RQM includes the following operators:

$$
\hat{M}_{I}, \hat{J}^{2}, \hat{J}_{3}, \hat{\mathbf{P}} .
$$

here $\hat{M}_{I}$ is mass operator, $\hat{J}^{2}$ is square of total angular momentum, $\hat{J}_{3}$ is projection of $\hat{J}$ on the quantization axis, $\hat{\mathbf{P}}$ is momentum operator.

In the instant form of dynamics $\hat{J}^{2}, \hat{J}_{3}, \hat{\vec{P}}$ operators coincide with non-interaction system ones, therefore their diagonalization is reduced to appropriate basis choice.

The mass operator of system with interaction is constructed by additive way:

$$
\hat{M}_{I}=\hat{M}_{0}+\hat{V},
$$

here $\hat{M}_{0}$-is non-interactions system invariant mass operator, $\hat{V}$ is operator of interaction.

On the operators $(1.1),(1.2)$ we impose the following additional conditions:

$$
\begin{gathered}
\hat{M}_{I}=\hat{M}_{0}^{+}, \hat{M}_{I}>0, \\
{[\hat{\vec{P}}, \hat{V}]=[\hat{\vec{J}}, \hat{V}]=\left[\hat{\vec{\nabla}}_{P}, \hat{V}\right]=0 .}
\end{gathered}
$$

So, the problem of calculation of wave function is reduces to the solution of eigenvalue problem for $M_{I}$ in appropriate basis:

$$
\hat{M}_{I}|\psi(a, b, c)\rangle=\left(\hat{M}_{0}+\hat{V}\right)|\psi(a, b, c)\rangle=\lambda|\psi(a, b, c)\rangle .
$$

where $\lambda$ is eigenvalue of mass operator, $a, b, c$ is indices of system components.

In the paper [4] derived a formula for calculation of the form factors of the composite system. For three-body system corresponding expression has following form:

$$
G_{E, M}\left(Q^{2}\right)=\sum_{\gamma \gamma^{\prime}} \int d \sqrt{s_{b c}} d \sqrt{s_{b c}^{\prime}} d \sqrt{s} d \sqrt{s^{\prime}} \varphi_{\gamma}^{J}\left(\sqrt{s}, \sqrt{s_{b c}}\right) F_{E, M}^{\gamma \gamma^{\prime}} \varphi_{\gamma^{\prime}}^{J^{\prime}}\left(\sqrt{s^{\prime}}, \sqrt{s_{b c}^{\prime}}\right),
$$

here $G_{E, M}\left(Q^{2}\right)$ is electric and magnetic form factors of three-body system in composite quark model, respectively. $F_{E, M}^{\gamma \gamma^{\prime}}$ is so called free three-body form factors and $\varphi_{\gamma}^{J}\left(\sqrt{s}, \sqrt{s_{b c}}\right)$ is wave function of the three-particle in RQM. Sense of other values will be clarified later.

The $F_{E, M}^{\gamma \gamma}$ will be calculated in our paper. 


\section{Construction of three body basis}

To obtain the free electromagnetic form factors of the three-body systems, we first need to construct a basis which will describe a composite particle. We will construct two bases that can be used to describe the three-body system.

The first basis consists of a direct product of one-particle vector of state with the vector of two-particle subsystems:

$$
\left|\vec{p}_{a}, m_{a} ; \vec{P}_{b c}, \sqrt{s_{b c}}, L_{b c}, S_{b c}, J_{b c}, m_{J_{b c}}\right\rangle=\left|\vec{p}_{a}, m_{a} ;\right\rangle \otimes\left|\vec{P}_{b c}, \sqrt{s_{b c}}, L_{b c}, S_{b c}, J_{b c}, m_{J_{b c}}\right\rangle .
$$

The vector of two-particle subsystem is constructed as a direct product of two single-particle vectors, and is associated with either as:

$$
\begin{gathered}
\left|\vec{P}_{b c}, \sqrt{s_{b c}}, L_{b c}, S_{b c}, J_{b c}, m_{J_{b c}}\right\rangle=\sum \int d \hat{k}_{b c}\left|\vec{p}_{b}, m_{b} ; \vec{p}_{c}, m_{c}\right\rangle Y_{L_{b c} m_{L_{b c}}}\left(\hat{k}_{b c}\right) \times \\
\times\left\langle 1 / 2,1 / 2, \tilde{m}_{b}, \tilde{m}_{c} \mid S_{b c} m_{S_{b c}}\right\rangle\left\langle S_{b c}, L_{b c}, m_{S_{b c}}, m_{L_{b c}} \mid J_{b c}, m_{J_{b c}}\right\rangle D_{m_{b}{ }^{\prime} m_{b}}^{1 / 2}\left(P_{b c} p_{b}\right) D_{m_{c}{ }^{\prime} m_{c}}^{1 / 2}\left(P_{b c} p_{c}\right),
\end{gathered}
$$

where $Y_{l m}(\hat{k})$ is spherical function, $\left\langle j_{1}, m_{1}, j_{2}, m_{2} \mid j, m\right\rangle$ is Clebsch-Gordan coefficient, $D_{m^{\prime} m}^{j}\left(p^{\prime} p\right)$ is Wigner matrix.

Normalization of single-particle vectors is well known:

$$
\left\langle\vec{p}_{a}^{\prime}, m_{a}^{\prime} \mid \vec{p}_{a}, m_{a}\right\rangle=2 p_{a 0} \delta\left(\vec{p}_{a}-\vec{p}_{a}^{\prime}\right) \delta_{m_{a} m_{a}^{\prime}}
$$

and the vectors of two-particle subsystems is normalized as follows:

$$
\begin{gathered}
\left\langle\vec{P}_{b c}^{\prime}, \sqrt{s_{b s}^{\prime}}, L_{b c}^{\prime}, S_{b c}^{\prime}, J_{b c}^{\prime}, m_{J_{b c}}^{\prime} \mid \vec{P}_{b c}, \sqrt{s_{b c}}, L_{b c}, S_{b c}, J_{b c}, m_{J_{b c}}\right\rangle= \\
=N^{2} 2 P_{b c}^{0} \delta\left(\vec{P}_{b c}-\vec{P}_{b c}^{\prime}\right) \delta\left(\sqrt{s_{b c}}-\sqrt{s_{b c}^{\prime}}\right) \delta_{m_{b c}} m_{J_{b c}}^{\prime} \delta_{S_{b c} S_{b c}^{\prime}} \delta_{L_{b c} L_{b c}^{\prime}} \delta_{J_{b c} J_{b c}^{\prime}} .
\end{gathered}
$$

Also we can construct a three-body basis with separated center-of-mass motion:

$$
\left|\vec{P}, m_{J} ; \sqrt{s}, \sqrt{s_{b c}}, L, S, J,(L, S, J)_{b c}\right\rangle .
$$

This basis can be expressed in terms of the basis (2.1) and basis (2.2):

$$
\begin{gathered}
\left|\vec{P} \sqrt{s}, L, S, J, m_{J} ; \sqrt{s_{b c}},(L, S, J)_{b c}\right\rangle=\sum\left|\vec{p}_{a}, m_{a} ; \vec{P}_{b c}, \sqrt{s_{b c}}, L_{b c}, S_{b c}, J_{b c}, m_{J_{b c}}\right\rangle \times \\
\times Y_{L m_{L}}(\hat{k})\left\langle 1 / 2, J_{b c}, m_{a}^{\prime}, m_{J_{b c}}^{\prime} \mid S, m_{S}\right\rangle\left\langle S, L, m_{S}, m_{L} \mid J, m_{J}\right\rangle D_{m_{a}{ }^{\prime} m_{a}}^{1 / 2}\left(P p_{a}\right) D_{m_{J_{b c}}{ }^{\prime} m_{b c}}^{J_{b c}}\left(P P_{b c}\right) .
\end{gathered}
$$

Vector $\left|\vec{P}, m_{J} ; \sqrt{s}, \sqrt{s_{b c}}, L, S, J,(L, S, J)_{2}\right\rangle$ is transformed by analogy to one of the single particle with momentum $\vec{P}$ and spin $\vec{J}$.

The wave function of the three-particle with interaction in RQM is presented by following way:

$$
\left\langle\vec{P} \sqrt{s}, L, S, J, m_{J} ; \sqrt{s_{b c}},(L, S, J)_{b c} \mid \vec{p}_{c}, \mu_{c}\right\rangle=N_{c} \delta\left(\vec{P}-\vec{p}_{c}\right) \delta_{m_{J} m_{c}} \varphi_{\gamma}^{J}\left(\sqrt{s}, \sqrt{s_{b c}}\right),
$$

here $\gamma \equiv\left\{L, S,(L, S, J)_{b c}\right\}, N_{c}$ is normalization factor. 


\section{Parametrization of the electromagnetic current operator}

Let us consider the calculation of the matrix element for the free electromagnetic current operator of three-particles system. We will use the method of invariant parametrization of local operators [4].

The electromagnetic current of single-particle system can be written as:

$$
\begin{gathered}
\left\langle\vec{p}, M, j, m\left|J_{\mu}(0)\right| \vec{p}^{\prime}, M, j, m^{\prime}\right\rangle=\sum_{m^{\prime \prime}}\left\langle m\left|D^{j}\left(p p^{\prime}\right)\left\{F_{1} K_{\mu}^{\prime}+F_{2} \Gamma_{\mu}\left(p^{\prime}\right)+F_{3} R_{\mu}+F_{4} K_{\mu}\right\}\right| m^{\prime}\right\rangle, \\
F_{k}=\sum_{n=0}^{2 j} f_{i k}\left(Q^{2}\right)\left(i p_{\mu} \Gamma^{\mu}\left(p^{\prime}\right)\right)^{k}
\end{gathered}
$$

here

$$
K_{\mu}^{\prime}=\left(p+p^{\prime}\right)_{\mu}, K_{\mu}=\left(p-p^{\prime}\right)_{\mu}=Q_{\mu}, R_{\mu}=\varepsilon_{\mu \nu \lambda \rho} p^{v} p^{\prime \rho} \Gamma^{\rho}\left(p^{\prime}\right),
$$

$\Gamma^{\mu}(p)$ is operator of the relativistic spin particle with momentum $p, \varepsilon_{\mu \nu \lambda \rho}$ is antisymmetric tensor, $f_{i k}\left(Q^{2}\right)$ is invariant form factor.

For a more improvements working with this parametrization, we impose a operator of additional conditions:

1. Condition of conservation:

$$
j_{\mu} K^{\mu}=j_{\mu} Q^{\mu}=0 \rightarrow F_{4}=0 .
$$

2. Self-conjugate condition:

$$
\begin{aligned}
\Gamma_{\mu}\left(p^{\prime}\right) \rightarrow & \Gamma_{\mu}\left(p^{\prime}\right)-\frac{K_{\mu}^{\prime}}{K^{\prime 2}}\left(p_{v} \Gamma^{v}\left(p^{\prime}\right)\right), n=0, \\
F_{k} A_{\mu} \rightarrow & \frac{1}{2}\left\{F_{k}, A_{\mu}\right\}_{+}, k=2,3, n \neq 0, \\
& F_{k} \rightarrow i F_{k}, k=3,4 .
\end{aligned}
$$

3. Condition of orthogonality of the four-vectors:

$$
\Gamma_{\mu}\left(p^{\prime}\right) \rightarrow \Gamma_{\mu}\left(p^{\prime}\right)-\left(\frac{K_{\mu}}{K^{2}}+\frac{K_{\mu}^{\prime}}{K^{\prime 2}}\right)\left(p_{v} \Gamma^{v}\left(p^{\prime}\right)\right) .
$$

4. The condition of parity conservation.

This condition is satisfied if all the terms in the sum (3.2) contain an even number of pseudovector factors $\Gamma^{\mu}\left(p^{\prime}\right)$. So, the sums in $F_{1}$ are over even $\mathrm{n}: 2 j \geq n \geq 0$, in $F_{2}$ over even $\mathrm{n}$ : $2 j-1 \geq n \geq 0$, and in $F_{3}$ over odd $\mathrm{n}: 2 j-1 \geq n>0$.

So, the parametrization has the form:

$$
\begin{gathered}
\left\langle\vec{p}, M, j, m\left|J_{\mu}(0)\right| \overrightarrow{p^{\prime}}, M, j, m^{\prime}\right\rangle=\sum_{m^{\prime \prime}}\left\langle m\left|D^{j}\left(p p^{\prime}\right)\right| m^{\prime \prime}\right\rangle \times \\
\times\left\langle m^{\prime \prime}\left|F_{1} K_{\mu}^{\prime}+\left\{F_{2}\left(\Gamma_{\mu}\left(p^{\prime}\right)-\left(\frac{K_{\mu}}{K^{2}}+\frac{K_{\mu}^{\prime}}{K^{\prime 2}}\right)\left(p_{v} \Gamma^{v}\left(p^{\prime}\right)\right)\right)\right\}_{+}+i\left\{F_{3} R_{\mu}\right\}_{+}\right| m^{\prime}\right\rangle .
\end{gathered}
$$


In case $j=\frac{1}{2}$ we obtain:

$$
\left\langle\vec{p}, M, \frac{1}{2}, m\left|J_{\mu}(0)\right| \vec{p}^{\prime}, M, \frac{1}{2}, m^{\prime}\right\rangle=\sum_{m^{\prime \prime}}\left\langle m\left|D^{\frac{1}{2}}\left(p p^{\prime}\right)\right| m^{\prime \prime}\right\rangle\left\langle m^{\prime \prime}\left|f_{10}\left(Q^{2}\right) K_{\mu}^{\prime}+i f_{30}\left(Q^{2}\right) R_{\mu}\right| m^{\prime}\right\rangle .
$$

The invariant form factors from (3.4) are connected with Sachs form factors:

$$
f_{10}\left(Q^{2}\right)=\frac{2 M G_{E}\left(Q^{2}\right)}{\sqrt{4 M^{2}+4 Q^{2}}}, f_{30}\left(Q^{2}\right)=-\frac{4 G_{M}\left(Q^{2}\right)}{M \sqrt{4 M^{2}+Q^{2}}}
$$

Let us consider now the electromagnetic current of the three-body system in basis (2.5). The current operator of the free three-particle system has the following form:

$$
J_{0 \mu}(0)=J_{\mu}^{a}(0) \otimes I^{b c} \oplus J_{\mu}^{b}(0) \otimes I^{a c} \oplus J_{\mu}^{c}(0) \otimes I^{a b} .
$$

here $J_{\mu}^{i}(0)$ - current operator of the single-particle.

The current matrix element in basis (2.1) has the form:

$$
\begin{aligned}
& \left\langle\vec{p}_{a}, m_{a} ; \vec{P}_{b c}, \sqrt{s_{b c}}, m_{J_{b c}}, \gamma_{b c}\left|J_{0 \mu(0)}\right| \vec{p}_{a}^{\prime}, m_{a}^{\prime} ; \vec{P}_{b c}^{\prime}, \sqrt{s_{b c}^{\prime}}, m_{J_{b c}}^{\prime}, \gamma_{b c}^{\prime}\right\rangle= \\
= & \sum_{P(a b c)}\left\langle\vec{P}_{b c}, \sqrt{s_{b c}}, m_{J_{b c}}, \gamma_{b c} \mid \vec{P}_{b c}^{\prime}, \sqrt{s_{b c}^{\prime}}, m_{J_{b c}}^{\prime}, \gamma_{b c}^{\prime}\right\rangle\left\langle\vec{p}_{a}, m_{a}\left|J_{\mu}^{a}\right| \vec{p}_{a}^{\prime}, m_{a}^{\prime}\right\rangle,
\end{aligned}
$$

here $\gamma_{b c} \equiv\left\{L_{b c}, S_{b c}, J_{b c}\right\}$. In the basis with separate the center-of-mass motion will have a parametrization similar to the one-particle formula (3.4):

$$
\begin{aligned}
& \left\langle\vec{P}, m_{J} ; \sqrt{s}, \sqrt{s_{b c}}, \gamma\left|J_{0 \mu(0)}\right| \vec{P}^{\prime}, m_{J}^{\prime} ; \sqrt{s^{\prime}}, \sqrt{s_{b c}^{\prime}}, \gamma\right\rangle= \\
= & \sum_{m_{J}^{\prime \prime}}\left\langle m_{J}\left|D^{1 / 2}\left(P P^{\prime}\right)\right| m_{J}^{\prime \prime}\right\rangle\left\langle m_{J}^{\prime \prime}\left|F_{E}^{\gamma \gamma^{\prime}} A_{\mu}^{1}+F_{M}^{\gamma \gamma^{\prime}} A_{\mu}^{3}\right| m_{J}^{\prime}\right\rangle,
\end{aligned}
$$

here

$$
A_{\mu}^{1}=\frac{1}{Q^{2}}\left(\left(s-s^{\prime}+Q^{2}\right) P_{\mu}+\left(s^{\prime}-s+Q^{2}\right) P_{\mu}^{\prime}\right), A_{\mu}^{3}=\frac{i R_{\mu}}{\sqrt{s^{\prime}}},
$$

$F_{E, M} \equiv F_{E, M}\left(s, s_{b c}, Q^{2}, s^{\prime}, s_{b c}^{\prime}\right)$ are electric and magnetic form factor of three-particle system with nucleon numbers.

\section{Free form factor of three-body system}

Using the parametrization of the free three-particle system written in the form (3.5) and (3.6), we can express electromagnetic form factors of the three-particle system in terms of single-particle form factors. So, corresponding electric and magnetic form factors will be:

$$
\begin{gathered}
F_{E}^{00}=\sum_{P(a b c)} \frac{3 Q^{2}\left(s+s^{\prime}+Q^{2}\right)}{2 \lambda^{\frac{3}{2}}\left(s, s^{\prime},-Q^{2}\right)} \frac{k_{b c}}{k k^{\prime}} \delta\left(\sqrt{s_{b c}}-\sqrt{s_{b c}^{\prime}}\right)\left[A\left(s, s^{\prime}, Q^{2}, M^{2}\right) f_{10}^{a}\left(Q^{2}\right) \operatorname{Cos} \frac{\omega 1+\omega 2}{2}-\right. \\
\left.-\frac{M}{2} B\left(s, s^{\prime}, s_{b c}, Q^{2}, M^{2}\right) f_{30}^{a}\left(Q^{2}\right) \operatorname{Sin} \frac{\omega 1+\omega 2}{2}\right]
\end{gathered}
$$




$$
\begin{gathered}
F_{M}^{00}=\sum_{P(a b c)} \frac{Q^{2}\left(s+s^{\prime}+Q^{2}\right)}{8 \lambda^{\frac{3}{2}}\left(s, s^{\prime},-Q^{2}\right)} \frac{k_{b c}}{k k^{\prime}} \delta\left(\sqrt{s_{b c}}-\sqrt{s_{b c}^{\prime}}\right) \times \\
\times\left[-f_{10}^{a}\left(Q^{2}\right) B_{1}\left(s, s^{\prime}, s_{b c}, Q^{2}, M^{2}\right) \operatorname{Sin} \frac{\omega_{1}+\omega_{2}}{2}+f_{30}^{a}\left(Q^{2}\right)\left(B_{1}\left(s, s^{\prime}, s_{b c}, Q^{2}, M^{2}\right) \operatorname{Cos} \frac{\omega_{1}+\omega_{2}}{2}+\right.\right. \\
\left.\left.+B_{2}\left(s, s^{\prime}, s_{b c}, Q^{2}, M^{2}\right) \operatorname{Sin} \frac{\omega_{1}+\omega_{2}}{2}\right)\right], \\
F_{E}^{11}=\sum_{P(a b c)} \frac{Q^{2}\left(s+s^{\prime}+Q^{2}\right)}{4 \lambda \frac{3}{2}\left(s, s^{\prime},-Q^{2}\right)} \frac{k_{b c}}{k k^{\prime}} \delta\left(\sqrt{s_{b c}}-\sqrt{s_{b c}^{\prime}}\right)\left[2 A ( s , s ^ { \prime } , s _ { b c } , Q ^ { 2 } , M ^ { 2 } ) f _ { 1 0 } ^ { a } ( Q ^ { 2 } ) \left\{\operatorname{Cos} \frac{\omega 1+\omega 2}{2}+\right.\right. \\
\left.\left.+2 \operatorname{Cos} \frac{\omega 1+\omega 2+2 \omega 3}{2}\right\}-\frac{M}{4} B\left(s, s^{\prime}, s_{b c}, Q^{2}, M^{2}\right) f_{30}^{a}\left(Q^{2}\right)\left\{\operatorname{Sin} \frac{\omega 1+\omega 2}{2}+2 \operatorname{Sin} \frac{\omega 1+\omega 2+2 \omega 3}{2}\right\}\right], \\
\times\left[\frac{F_{M}^{11}=\sum_{P(a b c)} \frac{\left(4 \operatorname{Cos} \omega_{3}-1\right) Q^{2}\left(s+s^{\prime}+Q^{2}\right)}{4 \lambda^{\frac{3}{2}}\left(s, s^{\prime},-Q^{2}\right)} \frac{k_{b c}}{k k^{\prime}} \delta\left(\sqrt{s_{b c}}-\sqrt{s_{b c}^{\prime}}\right) \times}{Q^{2}\left(s, s^{\prime}, s_{b c}, Q^{2}, M^{2}\right)+\lambda\left(s, s^{\prime},-Q^{2}\right)\left(1-\frac{B_{1}\left(s, s^{\prime}, s_{b c}, Q^{2}, M^{2}\right)}{s^{\prime} \sqrt{2}}\right)} f_{10}^{a}\left(Q^{2}\right) \operatorname{Cos} \frac{\omega 1+\omega 2}{2}+\right. \\
\left.+\frac{M B_{3}\left(s, s^{\prime}, s_{b c}, Q^{2}, M^{2}\right)\left(\lambda\left(s, s^{\prime},-Q^{2}\right)-2 s^{\prime} Q^{2}\right)}{8 \sqrt{s^{\prime}}} f_{30}^{a}\left(Q^{2}\right) \operatorname{Sin} \frac{\omega 1+\omega 2}{2}\right],
\end{gathered}
$$

where $\sum_{P(a b c)}$ need to sum over the permutation of indices of components of the system,

$$
\begin{gathered}
A\left(s, s^{\prime}, Q^{2}, M^{2}\right)=s+s^{\prime}+Q^{2}+2\left(M^{2}-s_{b c}\right), \\
B\left(s, s^{\prime}, s_{b c}, Q^{2}, M^{2}\right)=\left(-M^{2} \lambda\left(s, s^{\prime},-Q^{2}\right)+s s^{\prime} Q^{2}-s_{b c} Q^{4}-\left(s_{b c}-M^{2}\right) Q^{2}\left(s+s^{\prime}\right)+Q^{2}\left(s_{b c}-M^{2}\right)^{2}\right)^{1 / 2} \\
B_{1}\left(s, s^{\prime}, s_{b c}, Q^{2}, M^{2}\right)=\left(\lambda\left(s, s^{\prime},-Q^{2}\right)+\lambda\left(s_{b c}, M^{2}, Q^{2}\right)+\right. \\
\left.+\left(Q^{2}+M^{2}\right)\left(M^{2}+2 s\right)+2 M^{2}\left(Q^{2}-s^{\prime}\right)\right)^{1 / 2} \\
B_{2}\left(s, s^{\prime}, s_{b c}, Q^{2}, M^{2}\right)=\frac{s\left(s+s^{\prime}\right)+\left(s+Q^{2}\right)\left(Q^{2}-4 s_{b} c+6 M^{2}\right)+s^{\prime}\left(3 Q^{2}-s^{\prime}\right)}{4}- \\
-\frac{s^{\prime}\left(2 s_{b c}+4 M^{2}-Q^{2}\right)}{4}-\frac{\left(s+Q^{2}\right)^{3}-2\left(s_{b c}-M^{2}\right)\left(s+Q^{2}\right)^{2}}{4 s^{\prime}} \\
B_{3}\left(s, s^{\prime}, s_{b c}, Q^{2}, M^{2}\right)=s^{\prime}\left(s^{\prime}-2 s_{b c}+4 M^{2}\right)-\left(s+Q^{2}\right)^{2}+2\left(s+Q^{2}\right)\left(s_{b c}-M^{2}\right) . \\
\lambda(a, b, c)=a^{2}+b^{2}+c^{2}-2(a b+a c+b c) .
\end{gathered}
$$

\section{Conclusion}

In the framework of the instant form of the relativistic quantum mechanics the electromagnetic current matrix element for the free three-body system is constructed at conditions of conservation and Lorentz-covariance. The electromagnetic form factors for free system of three fermion with quantum numbers of nucleon are calculated.

This work was supported by the Scientific and Scientific-Pedagogical Specialists of Innovative Russia Program (Grant No. 1338). 


\section{References}

[1] BABAR Collaboration, Phys.Rev. D. 80, 052002 (2009).

[2] O. Gayou et al., Phys.Rev.C. 64, 038202 (2001); Phys.Rev.Lett. 88, 092301 (2002).

[3] A.F. Krutov, V.E. Troitsky, Phys. Rev. C 65, 04501 (2002).

[4] A.F. Krutov, V.E. Troitsky, Phys. Rev. C 68, 018501 (2003). 